\title{
Succinct Proofs of Primality for the Factors of Some Fermat Numbers
}

\section{By Richard P. Brent}

Abstract. We give short and easily verified proofs of primality for the factors of the Fermat numbers $F_{5}, F_{6}, F_{7}$ and $F_{8}$.

1. Introduction. The Fermat numbers $F_{k}=2^{2^{k}}+1$ are prime for $1 \leqslant k \leqslant 4$ and have exactly two prime factors for $5 \leqslant k \leqslant 8$. Here we give 'succinct' [7] and easily verified proofs of primality for the prime factors of $F_{k}, 5 \leqslant k \leqslant 8$. We assume that the primality of integers smaller than $10^{7}$ is easy to check [5].

To prove that an integer $p$ is prime, it is sufficient to find an integer $x$ such that

$$
x^{p-1}=1(\bmod p)
$$

and, for all prime divisors $q$ of $p-1$,

$$
x^{(p-1) / q} \neq 1 \quad(\bmod p)
$$

Then $x$ is a primitive root $(\bmod p)$. The difficulty in finding such proofs lies in factorizing $p-1$; see e.g. [4].

2. Proofs of Primality. In Table 1 we give the least positive primitive root $\left(\bmod p_{k}\right)$ and the complete factorization of $p_{k}-1$ for the primes $p_{k}$ listed in Table 2. Using Table 1 , it is easy to verify that $p_{20}, \ldots, p_{1}$ are in fact prime. Since

$$
\begin{aligned}
& F_{5}=641 \cdot 6700417 \quad \text { (Euler), } \\
& F_{6}=274177 \cdot p_{1} \quad \text { (Landry), } \\
& F_{7}=p_{2} \cdot p_{3} \quad \text { (Morrison and Brillhart [6]), }
\end{aligned}
$$

and

$$
F_{8}=p_{8} \cdot p_{9} \quad(\text { Brent and Pollard }[3])
$$

this completes the required primality proofs.

Received January 15, 1981.

1980 Mathematics Subject Classification. Primary 10-04, 10A25, 10A40; Secondary 10A05, 10A10, 65C05, 68-04.

Key words and phrases. Factorization, Fermat numbers, primality testing, primitive root, Monte Carlo methods. 
TABLE 1

Primitive roots and factorizations

\begin{tabular}{rrl}
\hline$k$ & primitive root $\left(\bmod p_{k}\right)$ & factorization of $p_{k}-1$ \\
\hline 1 & 3 & $2^{8} \cdot 5 \cdot 47 \cdot 373 \cdot 2998279$ \\
2 & 3 & $2^{9} \cdot p_{4}$ \\
3 & 21 & $2^{9} \cdot 3^{5} \cdot 5 \cdot 12497 \cdot p_{6}$ \\
4 & 2 & $2 \cdot 7 \cdot 449 \cdot p_{5}$ \\
5 & 6 & $2 \cdot 3^{3} \cdot 181 \cdot 1896229$ \\
6 & 2 & $2 \cdot 3 \cdot 2203 \cdot p_{7}$ \\
7 & 3 & $2^{3} \cdot 6939437$ \\
8 & 3 & $2^{11} \cdot 157 \cdot p_{10}$ \\
9 & 43 & $2^{11} \cdot 3 \cdot 5 \cdot 7 \cdot 13 \cdot p_{11} \cdot p_{12}$ \\
10 & 6 & $2^{6} \cdot 5 \cdot 719 \cdot 16747$ \\
11 & 17 & $2 \cdot 1789 \cdot 10079 \cdot 876769$ \\
12 & 11 & $2^{4} \cdot 3 \cdot 8861 \cdot p_{13} \cdot p_{14} \cdot p_{15}$ \\
13 & 2 & $2^{2} \cdot 7 \cdot 223 \cdot 1699$ \\
14 & 2 & $2 \cdot 3^{2} \cdot 16879 \cdot p_{16}$ \\
15 & 5 & $2 \cdot 20939 \cdot p_{18}$ \\
16 & 11 & $2 \cdot p_{17}$ \\
17 & 2 & $2 \cdot 13 \cdot 1604753$ \\
18 & 5 & $2^{2} \cdot 3^{2} \cdot p_{19}$ \\
19 & 3 & $2^{4} \cdot 5 \cdot 7 \cdot p_{20}$ \\
20 & 2 & $2 \cdot 23 \cdot 29^{2} \cdot 283$ \\
\hline
\end{tabular}

TABLE 2

Primes related to factors of Fermat numbers

\begin{tabular}{rl}
\hline$k$ & \\
\hline 1 & 67280421310721 \\
2 & 59649589127497217 \\
3 & 5704689200685129054721 \\
4 & 116503103764643 \\
5 & 18533742247 \\
6 & 733803839347 \\
7 & 55515497 \\
8 & 1238926361552897 \\
9 & 93461639715357977769163558199606896584051237541638188580280321 \\
10 & 3853149761 \\
11 & 31618624099079 \\
12 & 1057372046781162536274034354686893329625329 \\
13 & 10608557 \\
14 & 25353082741699 \\
15 & 9243081088796207 \\
16 & 83447159 \\
17 & 41723579 \\
18 & 220714482277 \\
19 & 6130957841 \\
20 & 10948139 \\
\hline
\end{tabular}


3. Comments. The larger factor $p_{9}$ of $F_{8}$ was first proved to be prime by $\mathrm{H}$. C. Williams, using the method of [8]. At that time the complete factorization of $p_{9}-1$ was not known.

To obtain Table 1 we had to factorize several large integers. All nontrivial factorizations given in Table 1 were obtained using the Monte Carlo method of [2], implemented with the MP package [1]. The most difficult factorizations were those of the 56-digit integer $p_{11} p_{12}$ and the 30-digit integer $p_{14} p_{15}$. The numbers of arithmetic operations required for these factorizations were approximately as predicted by the probabilistic analysis of [2].

Acknowledgement. We thank the Australian National University for the provision of computer time.

Department of Computer Science

Australian National University

Canberra, A.C.T. 2600, Australia

1. R. P. BRENT, "Algorithm 524: MP, A Fortran multiple-precision arithmetic package," ACM Trans. Math. Software, v. 4, 1978, pp. 71-81.

2. R. P. Brent, "An improved Monte Carlo factorization algorithm," BIT, v. 20, 1980, pp. 176-184.

3. R. P. Brent \& J. M. Pollard, "Factorization of the eighth Fermat number," Math. Comp., v. 36, 1981, pp. 627-630.

4. D. E. KNUTH, The Art of Computer Programming, Vol. 2, Addison-Wesley, Menlo Park, 1969, Sec. 4.5.4.

5. D. N. LeHMER, List of Prime Numbers from 1 to 10,006,721, Hafner, New York, 1956.

6. M. A. Morrison \& J. Brillhart, "A method of factoring and the factorization of $F_{7}$," Math. Comp., v. 29, 1975, pp. 183-208.

7. V. R. PratT, "Every prime has a succinct certificate," SIAM J. Comput., v. 4, 1975, pp. 214-220.

8. H. C. Williams \& J. S. JUDD, "Some algorithms for prime testing using generalized Lehmer functions," Math. Comp., v. 30, 1976, pp. 867-886. 